Advanced Studies in Pure Mathematics 26, 2000

Analysis on Homogeneous Spaces and Representation Theory of Lie Groups pp. $33-48$

\title{
Propriétés Asymptotiques des Groupes Linéaires (II)
}

\author{
Yves Benoist
}

\begin{abstract}
.
Asymptotic properties of linear groups (II)

Let $G=K \exp \left(\mathfrak{a}^{+}\right) K$ be a Cartan decomposition of a connected real linear semisimple Lie group and $m: G \rightarrow \mathfrak{a}^{+}$be the associated map. Let $\Gamma$ be a Zariski dense subgroup of $G$ and $\ell_{\Gamma}$ be the asymptotic cone to $m(\Gamma)$. This cone is convex and of non empty interior (cf [3]).

We show that $m(\Gamma)$ fills completely $\ell_{\Gamma}$ in the following sense: for every $\varepsilon>0$ and every closed cone $C$ such that $C-\{0\}$ is included in the interior of $\ell_{\Gamma}$, every point of $C$ outside a compact is at distance less than $\varepsilon$ from $m(\Gamma)$.
\end{abstract}

\section{$\S$ Résumé}

Soient $G=K \exp \left(\mathfrak{a}^{+}\right) K$ une décomposition de Cartan d'un groupe linéaire semisimple réel connexe, $m: G \rightarrow \mathfrak{a}^{+}$l'application correspondante, $\Gamma$ un sous-semigroupe Zariski dense de $G$ et $\ell_{\Gamma}$ le cône asymptote à $m(\Gamma)$. Ce cône est convexe et d'intérieur non vide (cf [3]).

Nous montrons en quel sens $m(\Gamma)$ remplit complètement ce cone $\ell_{\Gamma}$.

\section{$\S$ Introduction}

Soient $G$ un groupe linéaire réel semisimple connexe, $\mathfrak{g}$ son algèbre de Lie, $\mathfrak{a}$ un sous-espace de Cartan de $\mathfrak{g}, \mathfrak{a}^{+}$une chambre de Weyl fermée de $\mathfrak{a}$ et $K$ un sous-groupe compact maximal de $G$ pour lequel on a la décomposition de Cartan: $G=K \exp \left(\mathfrak{a}^{+}\right) K$. Notons $m$ et $\ell$ les applications de $G$ dans la chambre de Weyl $\mathfrak{a}^{+}$définies par les relations $g \in K \exp (m(g)) K$ et $\ell(g)=\lim _{n \rightarrow \infty} \frac{1}{n} m\left(g^{n}\right)$.

Par exemple, lorsque $G=S L(k, \mathbb{R})$, la chambre de Weyl $\mathfrak{a}^{+}$est l'ensemble des matrices diagonales de trace nulle dont les coefficients

Received December 1, 1997. 
sont rangés par ordre décroissant et $K$ est le groupe spécial orthogonal. Dans ce cas, les coefficients de $\ell(g)$ sont les logarithmes des modules des valeurs propres de $g$ et les coefficients de $m(g)$ sont les logarithmes des valeurs propres de la racine carrée de la matrice symétrique $g^{t} g$.

Soit $\Gamma$ un sous-semigroupe de $G$. L'image $m(\Gamma)$ contrôle une partie importante du comportement asymptotique de $\Gamma$. Par exemple, c'est elle qui permet de savoir si $\Gamma$ agit proprement sur un espace homogène de $G$ donné (voir [2] et [13]).

J'ai montré dans [3] que, lorsque $\Gamma$ est Zariski dense, le cône $\ell_{\Gamma}$ asymptote à $m(\Gamma)$ est le plus petit cône fermé contenant $\ell(\Gamma)$, que ce cône est convexe et d'intérieur non vide et que $m(\Gamma)$ reste à distance bornée de $\ell_{\Gamma}$.

Le but de cet article est de montrer qu'à l'inverse, $m(\Gamma)$ (resp. $\ell(\Gamma)$ ) remplit complètement l'intérieur de $\ell_{\Gamma}$ au sens suivant. Munissons l'espace vectoriel $\mathfrak{a}$ d'une norme $\|$.$\| et de la distance associée.$

Théorème Soient $G$ un groupe linéaire réel semisimple connexe et $\Gamma$ un sous-semigroupe Zariski dense de G. Pour tout $\eta>0$ et tout cône fermé $C$ tel que $C-\{0\}$ est inclus dans l'intérieur de $\ell_{\Gamma}$, il existe un compact en dehors duquel tout point de $C$ est à distance inférieure à $\eta$ d'un point de $m(\Gamma)$ (resp. $\ell(\Gamma))$.

Le point central de la démonstration de ce théorème est la proposition suivante.

Proposition Soient $G$ un groupe linéaire réel semisimple connexe et $\Gamma$ un sous-semigroupe Zariski dense de $G$. Alors le sous-groupe de $\mathfrak{a}$ engendré par $\ell(\Gamma)$ est dense.

Notons, pour $g$ dans $\mathrm{SL}(m, \mathbb{R}), \ell_{1}(g)$ le logarithme de son rayon spectral $\lambda_{1}(g)$. Y. Guivarch ([8]) a démontré en utilisant des arguments probabilistes que le sous-groupe de $\mathbb{R}$ engendré par $\ell_{1}(\Gamma)$ est dense. Nos énoncés sont donc des généralisations multidimensionnelles de ce résultat. L'intérêt de tels énoncés provient des théorèmes de renouvellement (voir [12]).

Nos démonstrations sont purement algébriques. Elles reprennent la stratégie utilisée dans [3] pour démontrer que $\ell_{\Gamma}$ est d'intérieur non vide.

Je remercie Y.Guivarc'h qui a suscité mon intérêt pour cette question. 


\section{§1. Proximalité}

Pour tout espace métrique $(X, d)$, tout point $x$ de $X$ et tous fermés $Y, Z$ de $X$, on note

$$
\begin{gathered}
\delta(x, Y)=\inf \{d(x, y) / y \in Y\}, \\
\delta(Y, Z)=\sup \{\delta(y, Z) / y \in Y\} \text { et } \\
d(Y, Z)=\sup (\delta(Y, Z), \delta(Z, Y))
\end{gathered}
$$

la distance de Hausdorff entre $X$ et $Y$.

Soient $V$ un $\mathbb{R}$-espace vectoriel de dimension finie, $X=\mathbb{P}(V)$ l'espace projectif de $V$. On munit $V$ d'une norme $\|$.$\| et on définit une distance$ $d \operatorname{sur} X$ par

$$
d\left(x_{1}, x_{2}\right)=\inf \left\{\left\|v_{1}-v_{2}\right\| / v_{i} \in x_{i} \text { et }\left\|v_{i}\right\|=1 \quad \forall i=1,2\right\} .
$$

Soit $g$ un élément de $\operatorname{End}(V)-0$. Notons $\mu_{1}(g)=\|g\|$ la norme de $g: \mu_{1}(g)=\sup \{\|g x\| /\|x\|=1\}, \lambda_{1}(g)=\lim _{n \rightarrow \infty} \mu_{1}\left(g^{n}\right)^{\frac{1}{n}}$ le rayon spectral de $g, m_{1}(g):=\log \mu_{1}(g)$ et $\ell_{1}(g):=\log \lambda_{1}(g)$. Un élément $g$ de $\operatorname{End}(V)-0$ est dit proximal s'il a une seule valeur propre $\alpha$ telle que $|\alpha|=\lambda_{1}(g)$ et si cette valeur propre est simple. Cette valeur propre $\alpha$ est alors réelle et on note $x_{g}^{+} \in X$ la droite propre correspondante. On note $v_{g}^{+}$un vecteur de $x_{g}^{+}$de norme $1, V_{g}^{<}$l'hyperplan $g$-invariant supplémentaire à $x_{g}^{+}$et $X_{g}^{<}:=\mathbb{P}\left(V_{g}^{<}\right)$.

Dans la définition ci-dessous, issue de [1], on impose un contrôle uniforme sur la proximalité. Le réel $r$ contrôle tout d'abord la "géométrie" de $g$, le réel $\varepsilon$ contrôle ensuite sa "dynamique". On fixe $0<\varepsilon \leq r$ et on note

$$
\begin{aligned}
b_{g}^{\varepsilon} & :=\left\{x \in X / d\left(x, x_{g}^{+}\right) \leq \varepsilon\right\} \text { et } \\
B_{g}^{\varepsilon} & :=\left\{x \in X / \delta\left(x, X_{g}^{<}\right) \geq \varepsilon\right\} .
\end{aligned}
$$

Définition 1.1. Soit $0<\varepsilon \leq r$. Un élément $g$ proximal est dit $(r, \varepsilon)$ proximal si $\delta\left(x_{g}^{+}, X_{g}^{<}\right) \geq 2 r, g\left(B_{g}^{\varepsilon}\right) \subset b_{g}^{\varepsilon}$ et la restriction de $g \grave{a} B_{g}^{\varepsilon}$ est $\varepsilon$-Lipschitzienne.

Nous utiliserons la version suivante du critère de proximalité de Tits

Lemme 1.2. Soient $g$ dans $\mathrm{GL}(V), x^{+}$dans $\mathbb{P}(V), Y$ un hyperplan de $\mathbb{P}(V)$ et $r \geq \varepsilon>0$. On note $b^{\varepsilon}:=\left\{x \in X / d\left(x, x^{+}\right) \leq \varepsilon\right\}$ et $B^{\varepsilon}:=\{x \in X / \delta(x, Y) \geq \varepsilon\}$. On suppose que $\delta\left(x^{+}, Y\right) \geq 6 r$, $g\left(B^{\varepsilon}\right) \subset b^{\varepsilon}$ et la restriction de $g$ à $B^{\varepsilon}$ est $\varepsilon$-Lipschitzienne.

Alors $g$ est $(2 r, 2 \varepsilon)$-proximal, $d\left(x_{g}^{+}, x^{+}\right) \leq \varepsilon$ et $d\left(X_{g}^{<}, Y\right) \leq \varepsilon$. 
Démonstration Voir [15] ou le lemme 6.2 de [2].

Le lemme suivant affirme que, pour un élément $(r, \varepsilon)$-proximal $g$, le rayon spectral $\lambda_{1}(g)$ est une bonne approximation de la norme $\mu_{1}(g)$.

Lemme 1.3. Soient $V$ un $\mathbb{R}$-espace vectoriel de dimension finie et $0<\varepsilon \leq r$. Il existe des constantes $\left.c_{r, \varepsilon} \in\right] 0,1[$ telles que, pour tout $r>0$, on a $\lim _{\varepsilon \rightarrow 0} c_{r, \varepsilon}=1$ et, pour toute transformation linéaire $g$ de $V(r, \varepsilon)$-proximale, on a

$$
c_{r, \varepsilon} \mu_{1}(g) \leq \lambda_{1}(g) \leq \mu_{1}(g) .
$$

Démonstration Cela résulte des deux faits suivants: a) Les ensembles

$P_{r, \varepsilon}:=\{g \in \operatorname{End}(V) / g$ est $(r, \varepsilon)-$ proximal et de norme 1$\}$ sont compacts (lemme 6.3 de [2]).

b) L'application $g \rightarrow \lambda_{1}(g)$ est continue et vaut 1 sur le compact $P_{r}:=\cap_{\varepsilon>0} P_{r, \varepsilon}$.

La proposition suivante donne une approximation de $\lambda_{1}(g)$ et de $\mu_{1}(g)$ lorsque $g$ est un mot dont les lettres sont des éléments $(r, \varepsilon)$ proximaux.

Soient $g, h$ deux éléments proximaux de $\operatorname{End}(V)$, on note $\beta=$ $\beta_{1}(g, h)$ le réel défini par l'égalité: $v_{h}^{+}-\beta v_{g}^{+} \in V_{g}^{<}$.

Soient $g_{1}, \ldots, g_{l}$ des éléments proximaux de $\operatorname{End}(V)$, on note $g_{0}=g_{l}$ et

$$
\nu_{1}\left(g_{1}, \ldots, g_{l}\right)=\prod_{1 \leq j \leq l}\left|\beta_{1}\left(g_{j-1}, g_{j}\right)\right| .
$$

Ce produit ne dépend pas des choix faits.

Lemme 1.4. Pour tout $0<\varepsilon \leq r$ il existe des constantes $C_{r, \varepsilon}>0$ telles que

$$
\lim _{\varepsilon \rightarrow 0} C_{r, \varepsilon}=1 \text {, pour tout } r>0,
$$

et, si $g_{1}, \ldots, g_{l}$ sont des transformations linéaires $(r, \varepsilon)$-proximales vérifiant (en notant $g_{0}=g_{l}$ )

$$
\delta\left(x_{g_{j-1}}^{+}, X_{g_{j}}^{<}\right) \geq 6 r \text { pour } j=1, \ldots, l .
$$

Alors, pour tout $n_{1}, \ldots, n_{l} \geq 1$, le produit $g=g_{l}^{n_{l}} \cdots g_{1}^{n_{1}}$ est $(2 r, 2 \varepsilon)$ proximal et on a

$$
C_{r, \varepsilon}^{-l} \nu_{1}\left(g_{l}, \ldots, g_{1}\right) \leq \frac{\lambda_{1}\left(g_{l}^{n_{l}} \cdots g_{1}^{n_{1}}\right)}{\lambda_{1}\left(g_{l}\right)^{n_{l}} \cdots \lambda_{1}\left(g_{1}\right)^{n_{1}}} \leq C_{r, \varepsilon}^{l} \nu_{1}\left(g_{l}, \ldots, g_{1}\right) \text { et }
$$




$$
C_{r, \varepsilon}^{-l} \nu_{1}\left(g_{l}, \ldots, g_{1}\right) \leq \frac{\left\|g_{l}^{n_{l}} \cdots g_{1}^{n_{1}}\right\|}{\lambda_{1}\left(g_{l}\right)^{n_{l}} \cdots \lambda_{1}\left(g_{1}\right)^{n_{1}}} \leq C_{r, \varepsilon}^{l} \nu_{1}\left(g_{l}, \ldots, g_{1}\right) .
$$

Ce lemme n'est qu'un raffinement de la proposition 6.4 de [2]. Nous détaillons cependant sa démonstration. Le lemme suivant nous sera utile.

Lemme 1.5. Pour tout $0<\varepsilon<r$, il existe des constantes $d_{r, \varepsilon}>0$ telles que, pour tout $r>0$, on a $\lim _{\varepsilon \rightarrow 0} d_{r, \varepsilon}=1$, et pour tout hyperplan $W$ de $V$ et tout triplets de points $v, v^{\prime}, w$ de $V$ de norme 1 vérifiant $\delta(k v, \mathbb{P}(W)) \geq r, \delta\left(k v^{\prime}, \mathbb{P}(W)\right) \geq r$ et $d\left(k v, k v^{\prime}\right)<\varepsilon$ les nombres $\alpha \in k$ et $\alpha^{\prime} \in k$ définis par $v-\alpha w \in W$ et $v^{\prime}-\alpha^{\prime} w \in W$ vérifient les inégalités $d_{r, \varepsilon}^{-1} \leq \frac{\left|\alpha^{\prime}\right|}{|\alpha|} \leq d_{r, \varepsilon}$.

Démonstration C'est une conséquence de la compacité de l'ensemble de tels quadruplets $\left(W, v, v^{\prime}, w\right)$ et de la continuité de l'application $\left(W, v, v^{\prime}, w\right) \rightarrow \frac{\alpha^{\prime}}{\alpha}$.

Démonstration de la proposition 1.4 Notons $x_{j}^{+}, v_{j}^{+}, X_{j}^{<}, V_{j}^{<}$, $B_{j}^{\varepsilon}, b_{j}^{\varepsilon}$ pour $x_{g_{j}}^{+}, v_{g_{j}}^{+}$, etc... Comme $g_{j}^{n}$ est $(r, \varepsilon)$-proximal, que $x_{g_{j}^{n}}^{+}=x_{j}^{+}$ et que $X_{g_{j}^{n}}^{<}=X_{j}^{<}$, on peut supposer $n_{j}=1$, pour tout $j=1, \ldots, l$.

On a l'inclusion $g_{1}\left(B_{1}^{\varepsilon}\right) \subset b_{1}^{\varepsilon} \subset B_{2}^{\varepsilon} \quad$ car $\delta\left(x_{1}^{+}, X_{2}^{<}\right) \geq 2 r \geq 2 \varepsilon$. De même, $g_{2} g_{1}\left(B_{1}^{\varepsilon}\right) \subset b_{2}^{\varepsilon}$ et, après itération, $g\left(B_{1}^{\varepsilon}\right) \subset b_{l}^{\varepsilon}$ et $\left.g\right|_{B_{1}^{\varepsilon}}$ est $\varepsilon$-Lipschitzienne. On peut appliquer le lemme 1.2 car $\delta\left(x_{l}^{+}, X_{1}^{<}\right) \geq 6 r$. On obtient que $g$ est $(2 r, 2 \varepsilon)$-proximal et que $x_{g}^{+} \in b_{l}^{\varepsilon}$.

Soient $w_{0}=v_{g}^{+}, y_{0}=x_{g}^{+}$et, pour $j=1, \ldots, l$,

$$
w_{j}=g_{j} w_{j-1} \text { et } y_{j}=g_{j} y_{j-1} \text {. }
$$

Par construction, on a

$$
\begin{gathered}
y_{j} \in b_{j}^{\varepsilon} \text { pour } j=0, \ldots, l \text { et } \\
w_{l}=\lambda_{1}(g) w_{0} .
\end{gathered}
$$

Soit $\alpha_{j} \in k$ le nombre défini par l'égalité, pour $j=1, \ldots, l$,

$$
w_{j-1}=\alpha_{j} v_{j}^{+} \text {modulo } V_{j}^{<} .
$$

Comme $\delta\left(y_{j-1}, X_{j}^{<}\right)>5 r$ et $\delta\left(x_{j}^{+}, X_{j}^{<}\right) \geq 2 r$, le lemme 1.5 prouve que

$$
d_{r, \varepsilon}^{-1}\left|\beta_{1}\left(g_{j}, g_{j-1}\right)\right| \leq \frac{\left|\alpha_{j}\right|}{\left\|w_{j-1}\right\|} \leq d_{r, \varepsilon}\left|\beta_{1}\left(g_{j}, g_{j-1}\right)\right| .
$$


On a aussi

$$
w_{j}=\alpha_{j} \lambda_{1}\left(g_{j}\right) v_{j}^{+} \text {modulo } V_{j}^{<} .
$$

Comme $\delta\left(y_{j}, X_{j}^{<}\right) \geq r$, le même lemme 1.5 prouve que

$$
d_{r, \varepsilon}^{-1} \leq \frac{\left|\alpha_{j}\right| \lambda_{1}\left(g_{j}\right)}{\left\|w_{j}\right\|} \leq d_{r, \varepsilon}
$$

Ces deux inégalités donnent

$$
d_{r, \varepsilon}^{-2}\left|\beta_{1}\left(g_{j}, g_{j-1}\right)\right| \leq \frac{\left\|w_{j}\right\|}{\left\|w_{j-1}\right\|} \lambda_{1}\left(g_{j}\right)^{-1} \leq d_{r, \varepsilon}^{2}\left|\beta_{1}\left(g_{j}, g_{j-1}\right)\right| .
$$

En faisant le produit de ces $l$ inégalités et en remarquant que $\frac{\left\|w_{l}\right\|}{\left\|w_{0}\right\|}=$ $\lambda_{1}(g)$, on obtient

$$
d_{r, \varepsilon}^{-2 l} \nu_{1}\left(g_{l}, \ldots, g_{1}\right) \leq \frac{\lambda_{1}(g)}{\lambda_{1}\left(g_{l}\right) \cdots \lambda_{1}\left(g_{1}\right)} \leq d_{r, \varepsilon}^{2 l} \nu_{1}\left(g_{l}, \ldots, g_{1}\right) .
$$

puis à l'aide du lemme 1.3

$$
d_{r, \varepsilon}^{-2 l} \nu_{1}\left(g_{l}, \ldots, g_{1}\right) \leq \frac{\left\|g_{l} \cdots g_{1}\right\|}{\lambda_{1}\left(g_{l}\right) \cdots \lambda_{1}\left(g_{1}\right)} \leq d_{r, \varepsilon}^{2 l} c_{2 r, 2 \varepsilon}^{-1} \nu_{1}\left(g_{l}, \ldots, g_{1}\right) .
$$

Ce qui prouve notre proposition avec $C_{\varepsilon}=d_{r, \varepsilon}^{2} c_{2 r, 2 \varepsilon}^{-1}$.

Le corollaire suivant donne une autre définition de $\nu_{1}\left(g_{1}, \ldots, g_{l}\right)$.

Corollaire 1.6. Soient $g_{1}, \ldots, g_{l}$ des transformations linéaires proximales et $g_{0}=g_{l}$. On suppose que, pour tout $j=1, \ldots, l, x_{g_{j}}^{+} n^{\prime}$ est pas dans $X_{g_{j-1}}^{<}$. Alors on a l'égalité:

$$
\lim \frac{\lambda_{1}\left(g_{1}^{n_{1}} \cdots g_{l}^{n_{l}}\right)}{\lambda_{1}\left(g_{1}\right)^{n_{1}} \cdots \lambda_{1}\left(g_{l}\right)^{n_{l}}}=\lim \frac{\mu_{1}\left(g_{1}^{n_{1}} \cdots g_{l}^{n_{l}}\right)}{\lambda_{1}\left(g_{1}\right)^{n_{1}} \cdots \lambda_{1}\left(g_{l}\right)^{n_{l}}}=\nu_{1}\left(g_{1}, \ldots, g_{l}\right)
$$

où ces limites sont prises quand les entiers $n_{j}$ tendent tous vers $+\infty$

La définition suivante est motivée par le lemme 1.4.

Définition 1.7. Soient $0<\varepsilon \leq r$. Un sous-semigroupe $\Gamma$ de $\mathrm{GL}(V)$ est dit fortement $(r, \varepsilon)$-Schottky dans $\mathbb{P}(V)$ si

i) pour tout $h$ dans $\Gamma, h$ est $(r, \varepsilon)$-proximal,

ii) pour tout $h, h^{\prime}$ dans $\Gamma, \delta\left(x_{h}^{+}, X_{h^{\prime}}^{<}\right) \geq 6 r$. 


\section{§2. Représentations de G}

Soient $G$ un groupe linéaire réel semisimple connexe, $\mathfrak{g}$ son algèbre de Lie, Ad l'application adjointe, $\mathfrak{a}$ un sous-espace de Cartan de $\mathfrak{g}$ et $A_{G}$ le sous-groupe connexe de $G$ d'algèbre de Lie $\mathfrak{a}$. L'application exponentielle exp est une bijection de $\mathfrak{a}$ sur $A_{G}$. On note log la bijection inverse.

Pour tout caractère $\chi$ de $A_{G}$, on note $d \chi: \mathfrak{a} \rightarrow \mathbb{R}$ sa différentielle et $\mathfrak{g}_{\chi}:=\left\{X \in \mathfrak{g} / \forall a \in A_{G}, \operatorname{Ad}(a) X=\chi(a) X\right\}$ l'espace propre correspondant. On note $\Sigma:=\left\{\chi \neq 1 / \mathfrak{g}_{\chi} \neq 0\right\}$ l'ensemble des racines restreintes de $G, \Pi \subset \Sigma$ un système de racines simples, $A^{+}=\exp \left(\mathfrak{a}^{+}\right)$ la chambre de Weyl de $G$, elle est définie par $A^{+}=\left\{a \in A_{G} / \forall \chi \in \Pi, \chi(a) \geq 1\right\}$.

Soit $K$ un sous-groupe compact maximal de $G$ pour lequel on a la décomposition de Cartan $G=K A^{+} K$. Pour $g$ dans $G$, on note $\mu(g)=$ $\exp (m(g))$, l'élément de $A^{+}$tel que $g \in K \mu(g) K$ et $\lambda(g)=\exp (\ell(g))$ l'élément de $A^{+}$qui est conjugué à la composante hyperbolique de la décomposition de Jordan de $g$.

Soit $(V, \rho)$ une représentation de $G$ dans un $\mathbb{R}$-espace vectoriel de dimension finie. Pour tout caractère $\chi$ de $A_{G}$, on note $V_{\chi}:=\{v \in$ $\left.V / \forall a \in A_{G}, \rho(a) v=\chi(a) v\right\}$ l'espace propre correspondant. On note $\Sigma(\rho):=\left\{\chi / V_{\chi} \neq 0\right\}$ l'ensemble des poids restreints de $V$. On a $V=\bigoplus_{\chi \in \Sigma(\rho)} V_{\chi}$. On munit $\Sigma(\rho)$ de l'ordre défini par:

$\chi_{1} \leq \chi_{2} \Longleftrightarrow \chi_{1}(a) \leq \chi_{2}(a)$ pour tout $a$ dans $A^{+}$.

On suppose $\rho$ irréductible. L'ensemble $\Sigma(\rho)$ a alors un unique élément $\lambda$ maximal pour cet ordre appelé le plus haut poids restreint de $V$. La représentation $\rho$ est dite proximale si $\operatorname{dim} V_{\lambda}=1$. C'est toujours le cas lorsque $G$ est déployé.

Lemme 2.1. Soit $G$ un groupe linéaire réel semisimple connexe. Il existe $r$ représentations irréductibles proximales $\rho_{i}$ de $G$ dans des $\mathbb{R}$ espaces vectoriels $V_{i}$ de plus haut poids restreints $\left(\chi_{i}\right)_{1 \leq i \leq r}$ telles que l'application $a \rightarrow\left(\chi_{1}(a), \ldots, \chi_{r}(a)\right)$ est un isomorphisme de groupes de $A_{G}$ sur $] 0, \infty\left[{ }^{r}\right.$.

Démonstration C'est une conséquence de la classification des représentations de dimension finie de $G$.

Le lemme suivant de [2] relie les applications $\lambda$ et $\mu$ aux normes et rayons spectraux dans les représentations de $G$.

Lemme 2.2. $\quad$ Soit $G$ un groupe linéaire réel semisimple connexe. Pour toute représentation irréductible $(V, \rho)$ de $G$ de plus haut poids restreint 
$\chi$, il existe une norme euclidienne sur $V$, telle que, pour tout $g$ dans $G$, on $a$
a) $\lambda_{1}(\rho(g))=\chi(\lambda(g))$
b) $\mu_{1}(\rho(g))=\chi(\mu(g))$

Démonstration Choisissons une norme euclidienne $K$-invariante sur $V$ de sorte que, pour $a$ dans $A^{+}, \rho(a)$ soit un endomorphisme symétrique. C'est possible. On a alors

$$
\lambda_{1}(\rho(a))=\mu_{1}(\rho(a))=|\chi(a)| .
$$

Donc

$$
\lambda_{1}(\rho(g))=\lambda_{1}(\rho(\lambda(g)))=\chi(\lambda(g))
$$

et

$$
\mu_{1}(\rho(g))=\mu_{1}(\rho(\mu(g)))=|\chi(\mu(g))|
$$

\section{$\S$ 3. Produit d'éléments loxodromiques}

Définition 3.1. Un élément $g$ de $G$ est dit loxodromique (ou $\mathbb{R}$-régulier) si $\ell(g)$ est dans l'intérieur de la chambre de Weyl.

Il est équivalent de dire que, pour tout $i=1, \ldots, r$, l'élément $\rho_{i}(g)$ est proximal.

Définition 3.2. Un élément proximal $g$ de $G$ est dit $(r, \varepsilon)$-loxodromique si, pour tout $i=1, \ldots, r$, les images $\rho_{i}(g)$ sont $(r, \varepsilon)$-proximales

Définition 3.3. Soient $G$ un groupe linéaire réel semisimple connexe, $0<\varepsilon \leq r$ et $l \geq 2$. Soient $g_{1}, \ldots, g_{l}$ des éléments loxodromiques de $G$ et $g_{0}=g_{l}$. On suppose que $x_{\rho_{i}\left(g_{j}\right)}^{+} \notin X_{\rho_{i}\left(g_{j-1}\right)}^{<}$pour $j=1, \ldots, l$ et $i=1, \ldots, r$. On note alors $\nu=\nu\left(g_{1}, \ldots, g_{l}\right)$ l'élément de $A_{G}$ tel que: $\chi_{i}(\nu)=\nu_{1}\left(\rho_{i}\left(g_{1}\right), \ldots, \rho_{i}\left(g_{l}\right)\right)$, pour $i=1, \ldots, r$.

Notons e l'élément neutre de $G$. Les lemmes 1.4 et 2.2 donnent immédiatement:

Lemme 3.4. Soient $G$ un groupe linéaire réel semisimple connexe. Pour tout $0<\varepsilon \leq r$ il existe des compacts $N_{r, \varepsilon}$ de $\mathfrak{a}$ tels que

$$
\lim _{\varepsilon \rightarrow 0} N_{r, \varepsilon}=\{e\}, \text { pour tout } r>0,
$$

et, si $g_{1}, \ldots, g_{l}$ sont des éléments $(r, \varepsilon)$-loxodromiques vérifiant (en notant $g_{0}=g_{l}$ )

$$
\delta\left(x_{\rho_{i}\left(g_{j}\right)}^{+}, X_{\rho_{i}\left(g_{j-1}\right)}^{<}\right) \geq 6 r \text { pour } j=1, \ldots, l \text { et } i=1, \ldots, r .
$$


Alors, pour tout $n_{1}, \ldots, n_{l} \geq 1$, le produit $g=g_{1}^{n_{1}} \cdots g_{l}^{n_{l}}$ est $(2 r, 2 \varepsilon)$ loxodromique et on a

$$
\begin{aligned}
& \frac{\lambda\left(g_{1}^{n_{1}} \cdots g_{l}^{n_{l}}\right)}{\lambda\left(g_{1}\right)^{n_{1}} \cdots \lambda\left(g_{l}\right)^{n_{l}}} \in \nu\left(g_{1}, \ldots, g_{l}\right) N_{r, \varepsilon}^{l} \text { et } \\
& \frac{\mu\left(g_{1}^{n_{1}} \cdots g_{l}^{n_{l}}\right)}{\lambda\left(g_{1}\right)^{n_{1}} \cdots \lambda\left(g_{l}\right)^{n_{l}}} \in \nu\left(g_{1}, \ldots, g_{l}\right) N_{r, \varepsilon}^{l} .
\end{aligned}
$$

Le corollaire suivant est analogue au corollaire 1.6. Il prouve que $\nu\left(g_{1}, \ldots, g_{l}\right)$ ne dépend pas du choix des représentations $\rho_{i}$.

Corollaire 3.5. Soient $g_{1}, \ldots, g_{l}$ des éléments loxodromiques de $G$ comme dans la définition 3.3. On a les égalités:

$$
\lim \frac{\lambda\left(g_{1}^{n_{1}} \cdots g_{l}^{n_{l}}\right)}{\lambda\left(g_{1}\right)^{n_{1}} \cdots \lambda\left(g_{l}\right)^{n_{l}}}=\lim \frac{\mu\left(g_{1}^{n_{1}} \cdots g_{l}^{n_{l}}\right)}{\lambda\left(g_{1}\right)^{n_{1}} \cdots \lambda\left(g_{l}\right)^{n_{l}}}=\nu\left(g_{1}, \ldots, g_{l}\right)
$$

où ces limites sont prises quand les entiers $n_{j}$ tendent tous vers $+\infty$

Démonstration Cela résulte de la proposition 3.4 et du fait que, on peut fixer $r$ tel que, pour tout $\varepsilon$, il existe un entier $n_{0}$ tel que pour $n_{j} \geq n_{0}$ les éléments $g_{j}^{n_{j}}$ vérifient les hypothèses de la proposition 3.4. $\diamond$

Lemme 3.6. Soient $g$ un élément loxodromique de $G$ et $\Omega_{0}$ l'ouvert de $G$ sur lequel la fonction $h \rightarrow \theta(h):=\nu\left(g, h g h^{-1}\right)$ est définie. Alors, pour tout voisinage $\Omega$ de e dans $\Omega_{0}$, l'image $\theta(\Omega)$ est d'intérieur non vide dans $A_{G}$.

Démonstration Quitte à conjuguer $g$, on peut supposer que la composante hyperbolique de $g$ est dans l'intérieur de $A^{+}$.

Pour $i=1, \ldots, r$, notons $x_{i}^{+}$l'attracteur de $\rho_{i}(g)$ dans $X_{i}:=\mathbb{P}\left(V_{i}\right)$ et $V_{i}^{<}$le supplémentaire $g$-invariant. Choisissons un vecteur non nul $v_{i}^{+}$ de $x_{i}^{+}$et une forme linéaire $f_{i}^{<}$sur $V_{i}$ de noyau $V_{i}^{<}$. Le vecteur $v_{i}^{+} \in V_{i}$ est un vecteur de plus haut poids de la représentation $\rho_{i}$ tandis que $f_{i}^{<} \in V_{i}^{*}$ est un vecteur de plus bas poids de la représentation duale. On a les égalités

$$
\left(\chi_{i} \circ \theta\right)(h)=\nu_{1}\left(\rho_{i}(g), \rho_{i}\left(h g h^{-1}\right)\right)=\frac{\left|f_{i}^{<}\left(h v_{i}^{+}\right) f_{i}^{<}\left(h^{-1} v_{i}^{+}\right)\right|}{f_{i}^{<}\left(v_{i}^{+}\right)^{2}}
$$

On vérifie aisément que la différentielle en $e$ de l'application $\theta$ est nulle. Le hessien $\theta^{\prime \prime}$ de $\theta$ en $e$ est une forme bilinéaire symétrique sur 
$\mathfrak{g}$ à valeur dans $\mathfrak{a}$. Pour conclure, il suffit de montrer que l'image de ce hessien $\theta^{\prime \prime}$ engendre $\mathfrak{a}$.

Pour chaque racine simple $\alpha \in \Pi$, choisissons un triplet non nul $\left(Y_{\alpha}, H_{\alpha}, X_{\alpha}\right)$ tel que

$$
\begin{gathered}
Y_{\alpha} \in \mathfrak{g}_{-\alpha}, H_{\alpha} \in \mathfrak{a} \text { et } X_{\alpha} \in \mathfrak{g}_{\alpha} \\
{\left[H_{\alpha}, Y_{\alpha}\right]=-2 Y_{\alpha},\left[X_{\alpha}, Y_{\alpha}\right]=H_{\alpha} \text { et }\left[H_{\alpha}, X_{\alpha}\right]=2 X_{\alpha} .}
\end{gathered}
$$

L'existence de tels $s l_{2}$-triplets est bien connue et la famille $\left(H_{\alpha}\right)_{\alpha \in \Pi}$ est une base de $\mathfrak{a}$.

Un petit calcul basé sur les égalités

$$
Y_{\alpha} f_{i}^{<}=0, H_{\alpha} v_{i}^{+}=d \chi_{i}\left(H_{\alpha}\right) v_{i}^{+} \text {et } X_{\alpha} v_{i}^{+}=0
$$

donne le développement limité à l'ordre 2 suivant:

$$
\left(\chi_{i} \circ \theta\right)\left(e^{t X_{\alpha}} e^{s Y_{\alpha}}\right)=1+t s d \chi_{i}\left(H_{\alpha}\right)+o\left(t^{2}+s^{2}\right)
$$

On en déduit l'égalité $\theta^{\prime \prime}\left(X_{\alpha}, Y_{\alpha}\right)=H_{\alpha}$. Ce qui prouve bien que l'image du hessien engendre $\mathfrak{a}$.

\section{$\S 4$. Sous-semigroupes de Schottky}

La notion de sous-semigroupe $(r, \varepsilon)$-Schottky joue un rôle central dans notre démonstration. L'intérêt de ces semigroupes est que, d'une part, il est facile d'en construire dans tous les semigroupes Zariski denses (lemme 4.2) et que, d'autre part, on controle avec une bonne précision la projection de Cartan d'un tel semigroupe (lemme 3.4).

Définition 4.1. Soient $G$ un groupe linéaire réel semisimple connexe, $0<\varepsilon \leq r$. On dit qu'un sous-semigroupe $H$ de $G$ est fortement $(r, \varepsilon)-$ Schottky si, pour tout $i=1, \ldots, r$, le sous-semigroupe $\rho_{i}(H)$ est fortement $(r, \varepsilon)$-Schottky dans $\mathbb{P}\left(V_{i}\right)$.

Remarques - Tous les éléments de $H$ sont loxodromiques.

- Un sous-semigroupe d'un groupe fortement $(r, \varepsilon)$-Schottky est encore fortement $(r, \varepsilon)$-Schottky.

- On peut donc appliquer les estimations du lemme 3.4 à tous les mots dont les lettres sont dans $H$.

Avant de poursuivre rappelons quelques notations. Pour tout élément $g$ loxodromique de $G$, on note encore $x_{i}^{+}=x_{i, g}^{+}$l'attracteur de 
$\rho_{i}(g)$ dans $X_{i}:=\mathbb{P}\left(V_{i}\right), V_{i}^{<}=V_{i, g}^{<}$le supplémentaire $g$-invariant et $X_{i}^{<}=X_{i, g}^{<}:=\mathbb{P}\left(V_{i, g}^{<}\right)$. On fixe $0<\varepsilon \leq r$ où

$$
r=r(g):=\frac{1}{3} \inf _{1 \leq i \leq r} \delta\left(x_{i}^{+}, X_{i}^{<}\right)
$$

et on pose

$$
\begin{aligned}
& b_{i}^{\varepsilon}=\left\{x \in X_{i} / d\left(x, x_{i}^{+}\right) \leq \varepsilon\right\} \text { et } \\
& B_{i}^{\varepsilon}=\left\{x \in X_{i} / \delta\left(x, X_{i}^{<}\right) \geq \varepsilon\right\} .
\end{aligned}
$$

On note $G_{g}^{\varepsilon}:=$

$$
\left\{h \in G / \forall i h\left(B_{i}^{\varepsilon^{\prime}}\right) \subset b_{i}^{\varepsilon^{\prime}} \text { et }\left.h\right|_{B_{i}^{\varepsilon^{\prime}}} \text { est } \varepsilon^{\prime} \text {-Lipschitzien pour un } \varepsilon^{\prime}<\frac{\varepsilon}{2}\right\} .
$$

Par construction ce semigroupe $H=G_{g}^{\varepsilon}$ est ouvert. Par le critère de proximalité (lemme 1.2), $H$ est fortement $(r, \varepsilon)$-Schottky.

Lemme 4.2. Soient $G$ un groupe linéaire réel semisimple connexe et $\Gamma$ un sous-semigroupe Zariski dense de $G$. Alors, il existe un élément loxodromique $g_{0}$ de $\Gamma$ tel que, pour tout $\varepsilon<r\left(g_{0}\right)$, le semi groupe $G_{g_{0}}^{\varepsilon} \cap \Gamma$ est encore Zariski dense.

Démonstration Ce lemme n'est qu'une variante du théorème 6.4 de [3]. D'après l'appendice de [4], il existe un élément loxodromique $g_{0}$ dans $\Gamma$. Posons $H=G_{g_{0}}^{\varepsilon}$.

Soit $h$ un élément de $\Gamma$ tel que $h\left(x_{i}^{+}\right) \notin V_{i}^{<}$. Pour $n \gg 0$, l'élément $g_{0}^{n} h g_{0}^{n}$ est dans $\Gamma \cap H$. Comme l'adhérence de Zariski d'un semigroupe est un groupe, $h$ est dans l'adhérence de Zariski de $\Gamma \cap H$. Donc $\Gamma \cap H$ est Zariski dense dans $G$.

Lemme 4.3. Soient $G$ un groupe linéaire réel semisimple connexe et $g_{0}$ un élément loxodromique de G. Alors, pour tout voisinage ouvert $V$ de e dans $A_{G}$, il existe $\left.\varepsilon \in\right] 0, r\left(g_{0}\right)\left[\right.$ tel que pour tout $g_{1}, g_{2}$ dans le semigroupe $G_{g_{0}}^{\varepsilon}$ on a

$$
\frac{\lambda\left(g_{1} g_{2}\right)}{\lambda\left(g_{1}\right) \lambda\left(g_{2}\right)} \in V
$$

Démonstration Remarquons tout d'abord que, pour tout voisinage ouvert $W$ de $e$ dans $A_{G}$, il existe $\left.\varepsilon \in\right] 0, r\left(g_{0}\right)$ [ tel que pour tout $g_{1}, g_{2}$ dans $G_{g_{0}}^{\varepsilon}$ on a $\nu\left(g_{1}, g_{2}\right) \in W$. En effet, cela résulte de ce que, pour tout $g$ dans $G_{g_{0}}^{\varepsilon}$, on a

$$
d\left(x_{i, g}^{+}, x_{i, g_{0}}^{+}\right) \leq \varepsilon \text { et } d\left(X_{i, g}^{<}, X_{i, g_{0}}^{<}\right) \leq \varepsilon .
$$

Il suffit alors d'appliquer le lemme 3.4 en choisissant $W$ et $N_{r, \varepsilon}$ de sorte que $W N_{r \varepsilon}^{2} \subset V$. 


\section{$\S 5$. Semigroupes ouverts}

Voici encore quelques lemmes dont nous aurons besoin dans la démonstration de la proposition. Le premier permettra de supposer $\Gamma$ de type fini.

Lemme 5.1. Soit $\Gamma$ un sous-groupe Zariski dense d'un groupe linéaire réel semisimple connexe G. Alors il existe une famille finie $\gamma_{1}, \ldots, \gamma_{l}$ d'éléments de $\Gamma$ tels que, pour tout $n \geq 1$ le sous-groupe $\Gamma_{n}$ engendré par $\gamma_{1}^{n}, \ldots, \gamma_{l}^{n}$ est encore Zariski dense.

Démonstration C'est un exercice. On choisit les $\gamma_{j}$ de sorte que le groupe engendré par chacun d'eux est Zariski connexes et que le groupe engendré par tous ceux-ci est le groupe $R$ réunion des composantes Zariski connexes des adhérences de Zariski des sous-groupes de type fini de $\Gamma$. Par construction $R$ est distingué et l'image de $\Gamma$ dans le quotient $G / R$ est un groupe de torsion. Il contient donc, par le théorème de Schur un sous-groupe d'indice fini abélien (cf $[6])$. Donc $G / R$ est abélien et $G=R$.

Lemme 5.2. Soient $G$ un groupe linéaire réel semisimple connexe, $t \rightarrow \gamma_{j}^{t}$, pour $j=1, \ldots, s$, des groupes à un paramètre de $G$ et $\Delta$ le semigroupe engendré par $\gamma_{j}^{t}$ pour $t$ dans $[1, \infty[$ et $j=1, \ldots, s$. On suppose que $\Delta$ est Zariski dense dans $G$, alors $\Delta$ est d'intérieur non vide.

Démonstration C'est le lemme 7.1 de [3].

On appelle corps de Hardy un sous-corps stable par dérivation de l'anneau des germes en $+\infty$ de fonctions de classe $C^{\infty}$ sur $\mathbb{R}$ à valeurs réelles.

Rappel Toute solution d'une équation polynomiale (resp. d'une équation différentielle polynomiale du premier ordre ) à coefficients dans un corps de Hardy est encore dans un corps de Hardy. Voir, par exemple, l'article [14].

Définition 5.3. Soient $H$ un sous-semigroupe ouvert de $G$ et $\psi: H \rightarrow$ $\mathbb{R}$ une fonction analytique On note $(\star)$ la propriété suivante: pour tout groupe à un paramètre $t \rightarrow a^{t}$ d'éléments hyperboliques qui prend ses valeurs dans $H$ lorsque $t \geq 1$ et pour tous éléments $h_{1}, h_{2}$ de $H \cup\{1\}$, l'application $t \rightarrow \psi\left(h_{1} a^{t} h_{2}\right)$ est dans un corps de Hardy. 
Lemme 5.4. Soit $G$ un groupe linéaire réel semisimple connexe et $H$ un sous-semigroupe ouvert de $G$ dont tout élément est loxodromique et $\psi: H \rightarrow \mathbb{R}$ une fonction analytique qui vérifie la propriété $(\star)$. Soient $\gamma^{t}$ un groupe à un paramètre qui prend ses valeurs dans $H$ lorsque $t \geq 1$ et $h_{1}, h_{2}$ des éléments de $H \cup\{1\}$. On suppose que pour tout entier $t \geq 1$, on a l'égalité $\psi\left(h_{1} \gamma^{t} h_{2}\right)=0$.

Alors, cette égalité est encore vraie pour tout réel $t \geq 1$.

Démonstration C'est la même que celle du lemme 7.4 de [3].

\section{§6. Démonstrations}

\section{Démonstration de la proposition}

Il suffit de montrer que le seul caractère $\left.\chi: A_{G} \rightarrow\right] 0, \infty[$ tel que

$$
\log (\chi(\lambda(\Gamma))) \subset \mathbb{Z}
$$

est le caractère $\chi \equiv 1$.

On choisit alors $g_{0}$ comme dans le lemme 4.2 et on prend $\varepsilon$ suffisamment petit pour pouvoir choisir un voisinage ouvert $V$ de $e$ dans $A_{G}$ comme dans le lemme 4.3 tel que $V=V^{-1}$ et

$$
\log (\chi(V)) \subset]-1,1[\text {. }
$$

Le lemme 4.2, permet de supposer que $\Gamma$ est inclus dans le semigroupe ouvert $G_{g_{0}}^{\varepsilon}$.

On peut aussi supposer que $\Gamma$ est engendré par une famille finie d'éléments $\left(\gamma_{j}\right)_{1 \leq j \leq s}$ comme dans le lemme 5.1. Remarquons que chaque élément $\gamma_{j}$ est semisimple et notons $\gamma_{j}=m_{j} a_{j}=a_{j} m_{j}$ la décomposition de Jordan de $\gamma_{j}$ avec $m_{j}$ elliptique et $a_{j}$ hyperbolique. On note $t \rightarrow a_{j}^{t}$ le groupe à un paramètre d'éléments hyperboliques qui vérifient $a_{j}^{1}=a_{j}$.

L'adhérence du semigroupe engendré par $m_{j}$ est un groupe compact $M_{j}$. Quitte à remplacer $\gamma_{j}$ par une puissance, on peut supposer $M_{j}$ connexe. On note $t \rightarrow m_{j}^{t}$ un groupe à un paramètre d'éléments de $M_{j}$ tel que $m_{j}^{1}=m_{j}$ et on note $\gamma_{j}^{t}=m_{j}^{t} a_{j}^{t}=a_{j}^{t} m_{j}^{t}$. Quitte de nouveau à remplacer $\gamma_{j}$ par une puissance, on peut supposer que, pour tout $t \geq 1$, $M_{j} a_{j}^{t}$ est inclus dans $H$.

Notons $\Delta$ le sous-semigroupe de $H$ engendré par tous les éléments $\gamma_{j}^{t}$ avec $j=1, \ldots, s$ et $t \geq 1$. Ce semigroupe $\Delta$ contient $\Gamma$. Il est donc d'intérieur non vide (lemme 5.2).

Le lemme 3.6 prouve que l'ensemble $\{\nu(g, h) / g \in \Delta, h \in \Delta\}$ est d'intérieur non vide dans $A_{G}$. Le point b) du lemme suivant prouve alors que $\chi \equiv 1$. C'est ce que l'on voulait. 
Lemme 6.1. Gardons les notations ci-dessus.

a) L'application $\chi \circ \lambda$ est un morphisme de semigroupe de $\Delta$ dans ] $0, \infty[$.

b) Pour tout $g$, $h$ dans $\Delta$, On a $\chi(\nu(g, h))=1$

Démonstration a) Posons $\Phi(g)=\log (\chi(\lambda(g)))$. On veut montrer que, pour tout $g, h$ dans $\Delta$, on a l'égalité

$$
\Phi(g h)=\Phi(g)+\Phi(h)
$$

i) Montrons tout d'abord cette égalité lorsque $g$ et $h$ sont dans $\Gamma$. Dans ce cas, l'hypothèse (1) assure que $\Phi(g h), \Phi(g)$ et $\Phi(h)$ sont entiers. Le lemme 4.3 donne alors grace à l'inclusion (2)

$$
\Phi(g h)-\Phi(g)-\Phi(h) \in \log (\chi(V)) \cap \mathbb{Z}=\{0\} .
$$

ii) Montrons maintenant l'égalité (3) lorsque $g$ est dans $\Delta$ et $h$ est dans $\Gamma$. Remarquons tout d'abord que la fonction $\Phi: \Delta \rightarrow \mathbb{R}$ a la propriété $(\star)$. Cela résulte de notre rappel sur les corps de Hardy car il existe des réels $\beta_{i}$ tels que, pour tout $g$ dans $\Delta$, on a

$$
\Phi(g)=\sum_{i=1}^{r} \beta_{i} \ell_{1}\left(\rho_{i}(g)\right) .
$$

La fonction $\Psi: \Delta \rightarrow \mathbb{R}$ donnée par

$$
\Psi(g)=\Phi(g h)-\Phi(g)-\Phi(h)
$$

a aussi la propriété $(\star)$. Cette fonction $\Psi$ est nulle sur tous les mots

$$
g=\gamma_{j_{1}}^{t_{1}} \cdots \gamma_{j_{p}}^{t_{p}}
$$

où les exposants $t_{l} \geq 1$ sont entiers. Il résulte alors du lemme 5.4, et d'un raisonnement par récurrence que $\Psi$ est encore nulle sur ces mots lorsque les exposants $t_{l} \geq 1$ sont des réels. Donc $\Psi$ est nulle sur $\Delta$.

iii) L'égalité (3) lorsque $g$ et $h$ sont dans $\Delta$ se déduit de ii) par le même raisonnement appliqué à la fonction $h \rightarrow \Phi(g h)-\Phi(g)-\Phi(h)$.

b) Comme $\chi \circ \lambda$ est un morphisme de groupes, on a l'égalité, pour tout $n \geq 1$,

$$
\chi\left(\lambda\left(g^{n} h^{n}\right) \lambda(g)^{-n} \lambda(h)^{-n}\right)=1 .
$$

Prenons, à l'aide du corollaire 3.5, la limite lorsque $n$ tend vers $+\infty$ de cette égalité. On obtient $\chi(\nu(g, h))=1$.

Pour terminer la démonstration du théorème, nous aurons besoin du lemme suivant. 
Lemme 6.2. Soient $V$ un espace vectoriel normé, $\ell_{0}, \ldots, \ell_{t}$ des vecteurs de $V$,

$L:=\mathbb{R}^{+} \ell_{0}+\cdots+\mathbb{R}^{+} \ell_{t}, M:=\mathbb{Z} \ell_{0}+\cdots+\mathbb{Z} \ell_{t}, M^{+}:=\mathbb{N} \ell_{0}+\cdots+\mathbb{N} \ell_{t}$ et $\eta>0$. M.

On suppose que tout point de $V$ est à une distance inférieure à $\eta$ de

Alors tout point d'au moins un translaté $v_{0}+L$ de $L$ est à une distance inférieure à $\eta$ de $M^{+}$.

Démonstration Soit $K$ le compact $K:=\left\{\sum_{1 \leq j \leq t} x_{j} \ell_{j} / 0 \leq x_{j} \leq\right.$ 1 \}. On peut trouver une partie finie $F$ de $M$ telle que tout point de $\bar{K}$ est à une distance inférieure à $\eta$ d'un point de $F$. On choisit alors $v_{0}$ tel que

$$
v_{0}+F \subset M^{+} \text {. }
$$

Notre assertion résulte alors de l'égalité $L=K+M^{+}$.

Démonstration du théorème La proposition 4.3 de [3] prouve qu'il existe un entier $t>0$ et un réel $r>0$ tel que, pour tout $\varepsilon>0$, il existe des éléments $\gamma_{1}, \ldots, \gamma_{t}$ de $\Gamma$ qui engendrent un semigroupe $\Gamma^{\prime}$ Zariski dense et fortement $(r, \varepsilon)$-Schottky et tels que $C-\{0\}$ est encore inclus dans l'intérieur du cône

$$
L:=\mathbb{R}^{+} \ell\left(\gamma_{1}\right)+\cdots+\mathbb{R}^{+} \ell\left(\gamma_{t}\right) .
$$

Choisissons $\varepsilon$ suffisamment petit pour que le diamètre du compact $\log N_{r, \varepsilon}$ soit inférieur à $\frac{\eta}{2(t+1)}$. D'après la proposition, le sous-groupe de $\mathfrak{a}$ engendré par $\ell\left(\Gamma^{\prime}\right)$ est dense dans $\mathfrak{a}$. Il existe donc un élément $\gamma_{0}$ de $\Gamma^{\prime}$ tel que tout point de $\mathfrak{a}$ est à distance inférieure à $\frac{\eta}{2}$ du $\mathbb{Z}$-module

$$
M:=\mathbb{Z} \ell\left(\gamma_{0}\right)+\mathbb{Z} \ell\left(\gamma_{1}\right)+\cdots+\mathbb{Z} \ell\left(\gamma_{t}\right) .
$$

Notons $v_{1}:=\log \nu\left(\gamma_{0}, \gamma_{1}, \ldots, \gamma_{t}\right)$ et

$$
M^{+}:=\mathbb{N} \ell\left(\gamma_{0}\right)+\cdots+\mathbb{N} \ell\left(\gamma_{t}\right) .
$$

Le lemme 3.4 assure que tout point $v_{1}+n_{0} \ell\left(\gamma_{0}\right)+\cdots+n_{t} \ell\left(\gamma_{t}\right)$ de $v_{1}+M^{+}$ est à une distance inférieure à $\frac{\eta}{2}$ du point $\ell\left(\gamma_{1}^{n_{1}} \cdots \gamma_{t}^{n_{t}}\right)$ de $\ell(\Gamma)$ et du point $m\left(\gamma_{1}^{n_{1}} \cdots \gamma_{t}^{n_{t}}\right)$ de $m(\Gamma)$.

Le lemme 6.2 assure alors que tout point d'un translaté $v_{0}+v_{1}+L$ du cône $L$ est à distance inférieure à $\frac{\eta}{2}$ de $v_{1}+M^{+}$et est donc à distance inférieure à $\eta$ de $\ell(\Gamma)$ et de $m(\Gamma)$. Le complémentaire de $v_{0}+v_{1}+L$ dans $C$ est borné. Ceci prouve le théorème. 
Terminons cet article par une question: Un sous-groupe Zariski dense $\Gamma$ d'un groupe semisimple linéaire $G$ contient-il toujours une partie finie $F$ telle que $\ell(F)$ engendre un sous-groupe dense de $\mathfrak{a}$ ?

\section{Références}

[1] H. Abels, G. Margulis, G. Soifer - Semigroups containing proximal linear maps, Isr. Jour. Math. 91 (1995) p.1-30.

[2] Y. BENOIST - Actions propres sur les espaces homogènes réductifs, CRAS 319 (1994) p.937-940 et Annals of Math. 144 (1996) p.315-347.

[3] Y. Benoist - Propriétés asymptotiques des groupes linéaires, Geom. Funct. Anal. 7 (1997) p.1-47.

[4] Y. Benoist, F. LABourie - Sur les difféomorphismes d'Anosov affines à feuilletages stable et instable différentiables, Inv. Math. 111 (1993) p.285-308.

[5] N. Bourbaki - Groupes et algèbres de Lie, Paris (1975).

[6] C. CuRTis, I. REINER - Representation theory of finite groups and associative algebras, Interscience (1962).

[7] I. GOL'DShEID, G. MARGULIS - Lyapounov indices of a product of random matrices, Russ. Math. Surv. 44 (1989) p.11-71.

[8] Y. GuivarC'H - Communication orale.

[9] Y. Guivarc'H - Produits de matrices aléatoires et applications, Erg. Th. Dyn. Sys. 10 (1990), p.483-512.

[10] Y. Guivarc'H, G. Raugi - Propriétés de contraction d'un semigroupe de matrices inversibles, Isr. Jour. Math. 65 (1989) p.165-196.

[11] S. Helgason - Differential geometry, Lie groups and symmetric spaces, Acad. Press (1978).

[12] H. KESTEN - Random difference equations and renewal theory for products of random matrices, Acta Math. 131 (1973) p.207-248.

[13] T. KoBAYASHI - Criterion for proper actions on homogeneous spaces of reductive groups, Journal of Lie Theory 6 (1996) p.147-163.

[14] M. Rosenlicht - Hardy fields, Jour. Math. Anal. Appl. 93 (1983) p.297311.

[15] J. TITs - Free subgroups in linear groups, Jour. of Algebra 20 (1972) p.250-270.

CNRS-Ecole Normale Supérieure

45 rue d'Ulm, 75005 Paris

France

benoist@dmi.ens.fr 